\title{
Familial Periodic Paralyses
}

National Institute of Neurological Disorders and Stroke (NINDS)

\section{Source}

National Institute of Neurological Disorders and Stroke (NINDS). Familial Periodic

Paralyses.

Familial periodic paralyses are a group of inherited neurological disorders caused by mutations in genes that regulate sodium and calcium channels in nerve cells. They are characterized by episodes in which the affected muscles become slack, weak, and unable to contract. Between attacks, the affected muscles usually work as normal.

The two most common types of periodic paralyses are:

Hypokalemic periodic paralysis is characterized by a fall in potassium levels in the blood. In individuals with this mutation attacks often beg in in adolescence and are triggered by strenuous exercise, high carbohydrate meals, or by injection of insulin, glucose, or epinephrine. Weakness may be mild and limited to certain muscle groups, or more severe and affect the arms and legs. Attacks may last for a few hours or persist for several days. Some patients may develop chronic muscle weakness later in life.

Hyperkalemic periodic paralysis is characterized by a rise in potassium levels in the blood. Attacks often begin in infancy or early childhood and are precipitated by rest after exercise or by fasting. Attacks are usually shorter, more frequent, and less severe than the hypokalemic form. Muscle spasms are common. 